\title{
ADVANCES IN UNDERSTANDING PROGNOSIS IN MYELOMA
}

Dean Smith ${ }^{1}$, Kwee Yong ${ }^{2}$

${ }^{1}$ Department of Haematology, City Hospital, Nottingham

${ }^{2}$ Department of Haematology, University College London

Correspondence should be addressed to:

Dr Dean Smith

Department of Haematology

City Hospital

Hucknall Road

Nottingham

NG5 1PB

deansmith@doctors.org.uk

Tel: 01159691169

Keywords: Multiple Myeloma, Prognostic Factors, Myeloma Therapy

Running Title: Advances Understanding Prognosis Myeloma 


\section{ADVANCES IN UNDERSTANDING PROGNOSIS IN MYELOMA}

In the last two decades outcomes in multiple myeloma (myeloma) have greatly improved, due to the introduction of newer, more effective therapies. This improvement is not uniform. Response to treatment and survival remains heterogeneous, with some patients living for 1-2 years whilst others are alive and progression free at 10 years. This variation in outcome is due to patient characteristics plus features intrinsic to the myeloma tumour. Alongside the introduction of novel therapies there has been a greater understanding of disease biology and mechanisms of resistance. This has led to an increase in the number of prognostic markers which can be used in myeloma. This is important not only for more accurate counselling of patients in terms of disease outcome, but also in paving the way for risk adapted therapy. Both newer and traditional prognostic markers need to be used in the context of planned therapy. Indeed, the prognostic value of certain markers varies according to which therapy the patient receives. As such, these prognostic factors will require constant re-evaluation as agents with new mechanisms of action are added into the myeloma treatment algorithm. This article summarises current concepts of prognostic markers in myeloma.

\section{PATIENT CHARACTERISTICS}

\section{Age and Frailty}

Age remains a strong prognostic factor in myeloma (1). Median OS is around 4-6 years for patients over the age of 65 , compared to around 10 years for younger patients. Increased frequency of comorbidities and (leading to) less intensive treatment approaches are generally reported as the main reasons why elderly patients have a poorer outcome. Older patients do not have a higher incidence of adverse cytogenetic abnormalities. In fact, an IFM study found that $t(4 ; 14)$ was less prevalent in myeloma patients $>66$ years, unlike the case for $\operatorname{del}(17 \mathrm{p})$ (2). Fitness for stem cell transplantation appears to be more important than chronological age (3). A recent prospective study of 56 patients similarly found that age (above or below 70) did not impact on outcome in elderly patients treated with upfront autologous stem cell transplantation (ASCT), while reducing the dose of melphalan to $140 \mathrm{mg} / \mathrm{m}^{2}$ did (4). However, in an IFM study of 2316 patients treated with induction therapy (VAD or bortezomib based induction) followed by single or double ASCT, survival in patients aged 60-66 was significantly shorter than those under 60 . The risk of death increased linearly with age, with an increased risk of $22 \%$ every ten years (1).

Among patients of the same age, physical and cognitive functions can vary greatly. The recently released International Myeloma Working Group (IMWG) frailty score uses 3 tools (Katz Activity of Daily Living, Lawton Instrumental Activity of Daily Living and the Charlston Comorbidity Index), combined with age, to categorise elderly patients into 3 groups: fit, intermediate-fitness and frail (5). These assessments were performed on 869 newly diagnosed elderly patients in 3 prospective trials, using novel agents. The 3 year PFS was $48 \%$ in patients categorised as fit, $41 \%$ in intermediate-fitness and $33 \%$ in frail. Higher rates of adverse events, drug discontinuation and/or dose reduction were seen with increasing frailty. Thus, the frailty score predicts mortality and toxicity risk in elderly patients with newly diagnosed myeloma.

\section{Mode of Presentation}

A Swedish population study of 15000 patients diagnosed with myeloma over a 30 year period found that a previous diagnosis of MGUS correlated with longer OS, leading the authors to 
conclude that earlier treatment in the prior MGUS population led to improved OS. Alternatively, patients who have stable MGUS for a long enough period for it to be detected may have more indolent disease (6). Presentation through the emergency route and a delay in diagnosis of over 6 months are reported to be associated with decreased overall survival (7).

\section{Renal Function}

Patients presenting with acute renal failure have a worse outcome compared to those with normal kidney function (8), with increased early mortality, approaching $30 \%$ in the first 2 months in some series (9). Prognosis is related to the severity of the renal impairment, such that patients requiring dialysis treatment had a median survival of under 12 months in one single centre study (10).

\section{DISEASE BIOLOGY}

This is reflected in the growth rate, the pattern of organ infiltration and other features that indicate tumour bulk.

\section{Proliferation}

Proliferation rate increases with relapsed disease and is one of the strongest adverse prognostic factors. In one study, patients with a proliferation index (PI) of 8\%, measured as Ki67 expression, had showed shorter survival (11). Despite this, Ki-67 has not been widely used to assess proliferation in myeloma (12). The plasma cell labelling index (PCLI) measures the percentage of cells in S phase, determined by fluorescent microscopy, and its association with poorer outcome has been known for some time (13). The percentage of cells in S phase which confers a poor prognosis is low (1-3\%), reflecting the low cell turnover in most myeloma tumours, although in new era with novel agents and increasingly effective regimens, the threshold may change. Recently, a high proliferation index (i.e. $>1 \%$ cells in $\mathrm{S}$ phase) determined by multiparameter flow cytometry (MFC) has been shown to be a prognostic tool, with median OS 66 months vs 93 months using a cut off of $\geq 1 \%$ versus $<1 \%$ S-phase plasma cells (14). A subgroup of patients with a proliferation index $\geq 3 \%$ had median OS 45 months. This study included newly diagnosed transplant eligible patients and further analysis suggested bortezomib may overcome the adverse prognosis of a high proliferation index.

A gene expression based proliferation index selected proliferating genes differentially expressed between proliferating cells (malignant plasma cells plus non-malignant plasmablasts) and nonproliferating cells (non-malignant plasma cells and memory B cells) (12). Proliferation determined this way was an independent, continuous prognostic variable for EFS and OS in newly diagnosed patients treated with high dose therapy and ASCT both in Germany and as part of Total Therapy 2 (TT2).

\section{Monoclonal Protein Isotype}

IgA myeloma is associated with a poorer prognosis than IgG isotype, thought to be due to the association between IgA myeloma and t(4;14) (15). IgD myeloma accounts for 1-2\% of all cases. It is associated with advanced disease at diagnosis, extramedullary involvement, renal failure, elevated LDH and Beta-2 microglobulin and shorter survival. Survival has improved with the use of novel agent therapy, but remains inferior to survival of IgG, IgA and light chain myeloma (16). IgE myeloma is rarer, with fewer than 50 cases reported. It presents with similar features to IgD myeloma and is associated with an increased incidence of primary plasma cell leukaemia and $t(11 ; 14)(16)$. 


\section{Extramedullary Disease}

Extramedullary disease (EMD) is more prevalent in genetically high risk myeloma and is associated with shorter PFS and OS, in the context of novel agents. The Arkansas group found that EMD detected prior to ASCT was associated with $31 \% 5$ year OS compared to $59 \%$ for patients without EMD (17). A prospective study in Italy found that EMD detected by PET/CT (6\% of patients at diagnosis) was associated with shorter PFS and OS (18). Similarly, a large prospective Spanish trial found EMD associated with significantly shorter OS (median not reached vs 46.7 months), although the CR rate was significantly higher in this group when bortezomib/thalidomide/dexamethasone induction was used rather than thalidomide/dexamethasone alone (19).

\section{Plasma Cell Leukaemia and circulating plasma cells}

Plasma cell leukaemia is the most aggressive variant of plasma cell dyscrasias. It is defined as $>20 \%$ plasma cells in the peripheral blood with an absolute plasma cell count $>2 \times 10^{9} / 1$, and classified as primary (presenting de novo), or secondary (leukemic transformation in relapsed/refractory disease). Both categories have with a poor outcome. Primary plasma cell leukaemia is associated with a higher prevalence of extramedullary disease, light chain disease, high proliferative index, elevated $\mathrm{LDH}$ and Beta- 2 microglobulin and plasmablastic morphology (20). There is an increased incidence of adverse risk genetic features such as hypodiploidy, 1q+ and del(1p). Del(17p) is reported in almost $50 \%$ of pPCL and $70 \%$ sPCL, compared to $10 \%$ in newly diagnosed myeloma. A higher prevalence of $\mathrm{t}(11 ; 14)$ is reported in pPCL compared to myeloma (20).

No prospective data has been published. In seven retrospective studies including 20 or more patients, without novel therapy, median survival ranged from 6.8 to 12.6 months with 5 year survival under $10 \%$ (20). Even with novel therapies, prognosis remains poor (20). In the transplant setting, 272 patients treated with ASCT had median OS 25.7 months (versus 62.2 months for patients with myeloma) (21) whilst 19 of 50 patients (39\%) who underwent allogeneic transplant were alive at 3 years (22). Patients with pCL have high response rates but a short duration of response (20), suggesting a possible role for maintenance therapy. Survival of patients with sPCL is generally shorter than those with pPCL. SPCL generally represents heavily pre-treated, end stage myeloma thus patients are often refractory to therapy at this point, or have a short duration of response.

When the number of circulating plasma cells does not meet the diagnostic criteria for PCL, their presence remains indicative of a more proliferative and aggressive disease process. Retrospective analysis of blood samples from newly diagnosed and relapsed patients at the Mayo clinic showed the presence of low levels of circulating plasma cells, detected by immunophenotpying $(>400$ clonal plasma cells per 150,000 events), was associated with shorter OS, despite not meeting the diagnostic criteria for PCL (23).

\section{Intracranial Disease}

Intracranial involvement in myeloma is seen in around $1 \%$ of cases and can occur due to osteodural or CNS disease (24). The former arises from medullary disease involving skull bones, the latter as intraparenchymal lesions with or without leptomeningeal disease with plasma cells in the CSF. Prognosis in patients with CNS disease is reported to be $<6$ months. Patients with osteodural disease have a better prognosis, but still inferior to those without intracranial disease (24). 


\section{Lactate Dehydrogenase}

A number of studies have shown the association between elevated serum LDH levels and increased tumour proliferation rate, presence of extramedullary disease and shorter survival. This was initially investigated in the pre-novel agent era, but remains true in the era of novel agents (25).

\section{GENETIC LESIONS}

Myeloma tumours fall broadly into two subgroups; hyperdiploid myeloma is characterised by trisomies of odd-numbered chromosomes whilst non-hyperdiploid myeloma has recurrent chromosomal translocations, resulting in dysregulation of an oncogene, juxtaposed to the immunoglobulin heavy chain $(\mathrm{IgH})$ enhancer region on chromosome 14. Both pathways lead, directly or indirectly, to over-expression of a D-type cyclin. These chromosomal abnormalities are present at the precursor stage of monoclonal gammopathy of unknown significance (MGUS), and subsequent somatic mutations, translocations, copy number and/or epigenetic changes drive the progression to symptomatic myeloma. Each genetic event can carry prognostic significance, although the use of novel therapies may alter the prognostic impact of some factors previously considered high risk in the context of traditional therapies. Table 1 lists the cytogenetic abnormalities with prognostic significance in myeloma along with their incidence at diagnosis (26, 27).

\section{IgH Translocations}

$\underline{t(4 ; 14)}$

Translocation $(4 ; 14)$ has been shown to confer poor prognosis is a number of studies $(28-30)$. The translocation simultaneously dysregulates both MMSET on the der(4) and FGFR3 on the der(14), resulting in overexpression in $100 \%$ and $80 \%$ of tumours with this translocation respectively (26). Cyclin D2 is also upregulated, by an unknown mechanism. There is evidence to suggest patients with $\mathrm{t}(4 ; 14)$ are a heterogeneous group. In an IFM study, patients with $\mathrm{t}(4 ; 14)$ and a high $\mathrm{Hb}$ and low Beta-2 microglobulin at diagnosis treated with autologous stem cell transplantation in the pre-novel agent era had an improved outcome, although worse than those patients without $t(4 ; 14)$ (31). Also, the survival of $t(4 ; 14)$ positive patients varied significantly depending on risk status as defined by gene expression profiling (32). The poor prognostic impact of $t(4 ; 14)$ may be partly overcome by the use of bortezomib induction therapy prior to ASCT (33), especially if followed by bortezomib-based consolidation post-ASCT, such as in the Cavo study (34), and in Total Therapy 3 (35). Thus increasing evidence indicates that the prognostic impact of $t(4 ; 14)$ may depend on the treatment regimen used. The Mayo clinic mSMART risk stratification model has downgraded $t(4 ; 14)$ from high risk to intermediate risk due to the improved outcomes with bortezomib based therapy (36).

$\underline{t(14 ; 16) \text { and } t(14 ; 20)}$

$\mathrm{t}(14 ; 16)$ results in over-expression of MAF, transcription factor for CCND2, and therefore overexpression of cyclin D2. Whilst $\mathrm{t}(14 ; 16)$ is generally considered to be a poor prognostic marker (29), there is relatively little data to confirm this. Studies conducted by Mayo clinic and UK MRC have both shown $\mathrm{t}(14 ; 16)$ to be an independent poor prognostic factor $(28,37)$, however, the number of patients with $\mathrm{t}(14 ; 16)$ was small and in Mayo study patients were treated in the prenovel agent era. Retrospective analysis of 2 IFM trials found similar outcomes in patients with or without $\mathrm{t}(14 ; 16)$ (38). Again, the number of patients in these trials carrying $\mathrm{t}(14 ; 16)$ was small. Over-expression of MAF/MAFB was associated with poorer outcome in Total Therapy 3 trial (VTD-PACE, tandem ASCT, VTD then TD maintenance) (35), suggesting t(14;16) would be a 
poor prognostic marker. $\mathrm{t}(14 ; 20)$ has a low prevalence $(1-1.5 \%)$, results in overexpression of transcription factor MAFB which upregulates cyclin D2, and is associated with shorter survival (39).

\section{$\underline{t(11 ; 14)}$}

$\mathrm{t}(11 ; 14)$ juxtaposes CCND1 to the $\mathrm{IgH}$ enhancer region, leading to direct over-expression of CCND1 and therefore cyclin D1. It is generally considered to confer standard risk (30), however, as with $\mathrm{t}(4 ; 14)$, there is heterogeneity within the group. Based on gene expression profiling, the prognosis of tumours over-expressing CCND1 (i.e. with $t(11 ; 14))$ differed whether they coexpressed CD20 or not. Those without CD20 expression showed a quicker and deeper response to therapy, as shown by higher CR rate, but a shorter duration of CR and lower 2 year OS rates whereas those expressing CCND1 and CD20 had a slower time to achieve CR, lower CR rate but a longer duration of CR and higher 2 year OS rates (29). Recent studies have suggested $t(11 ; 14)$ confers an intermediate outcome with novel agents (40) and, in the case of AL amyloid, a worse outcome if treated with bortezomib-based regimens (41).

\section{$\underline{t(6 ; 14)}$}

$\mathrm{t}(6 ; 14)$ leads to direct over-expression of CCND3 and therefore cyclin D3, is found in around 5\% of cases and confered standard risk prognosis in the UK MRC Myeloma IX trial, which included thalidomide based therapy (28).

\section{Secondary Translocations}

The most notable secondary translocation involves $8 \mathrm{q} 24$, found in almost half of myeloma patients. These translocations lead to over-expression of $M Y C$. Unlike primary translocations, the majority of $M Y C$ translocations do not involve an Ig locus (42), suggesting other mechanisms of activation. Patients carrying MYC translocations had a poorer outcome in the UK MRC Myeloma IX trial (42).

\section{Copy Number Abnormalities}

Hyperdiploidy (48-75 chromosomal copies) is characterised by trisomies of at least 3 odd numbered chromosomes, and is associated with a standard outcomes. Primary $\operatorname{IgH}$ translocations are rarely found in hyperdiploid myeloma but generally seen in the non-hyperdiploid subgroup ( $<48$ or $>75$ chromosomes) (26). Hypodiploidy has been shown to be an independent predictor for reduced OS (43). The presence of trisomy of at least one odd numbered chromosome was reported to abrogate the poor prognostic impact of adverse lesions $[\mathrm{t}(4 ; 14), \mathrm{t}(14 ; 16), \mathrm{t}(14 ; 20)$, and del $(17 \mathrm{p})]$ in newly diagnosed patients treated with a novel agent in a retrospective analysis at Mayo Clinic (44). These findings were not replicated in the prospective UK MRC Myeloma IX study where coexistent hyperdiploidy or $\mathrm{t}(11 ; 14)$ did not overcome the poor prognosis associated with adverse cytogenetics (45).

Copy number abnormalities involving chromosome 1, i.e. gain of chromosome 1q (1q+) and loss of chromosome $1 \mathrm{p}$, del $(1 \mathrm{p})$, frequently occur together and are both associated with a poorer outcome. $1 \mathrm{q}+$ is found in $35-40 \%$ of cases $(26,27)$, the frequency increasing with disease progression (46). 1q+ impacted on survival in a number of studies, in both transplant eligible and ineligible patents $(28,47,48)$ and is associated with other adverse lesions such as $t(4 ; 14)(49)$. The adverse impact of $1 \mathrm{q}+$ is not altered by the use of thalidomide or bortezomib upfront $(47,48)$. Patients with more than 3 copies of 1q have a worse outcome, suggesting a possible a dosage effect for the genes carried on $1 \mathrm{q}(47,50)$. 
Loss of $1 \mathrm{p}$ is found in around $10 \%$ of cases (51). At least 2 potential genes on chromosome $1 \mathrm{p}$ could be involved. CDKN2C encodes for the cyclin dependent kinase inhibitor p18, loss of which leads to increased proliferation and poor prognosis (26). Mapping of chromosome 1p deletions in myeloma has also found $F A M 46 C$ at $1 \mathrm{p} 12$ to be a region associated with poor survival (52). The adverse impact of del(1p) is largely described in the context of ASCT (52).

Del(17p), including the TP53 locus, is seen in around 10\% of untreated myeloma cases, and prevalence increases with disease progression (26). It is a strong poor prognostic factor in numerous different treatment regimens $(30,33)$, however, data from the HOVON/GMMG-HD4 trial suggest that proteasome inhibitor triplet combination induction therapy, consolidated by double ASCT and maintenance with proteasome inhibitors can at least partly overcome the poor prognosis of del(17p) (47). The percentage of cells carrying del(17p) may be important, and a $60 \%$ cut-off has been suggested by IFM (33) whilst further analysis is suggested by the European Myeloma Network (53).

$\operatorname{Del}(13 q)$ is found in around $40 \%$ of cases, and the resulting haplo-insufficiency of $R B 1$, releasing the brake on the cell cycle, may be important (26). Detection of del(13q) by FISH alone is not an independent prognostic marker, as it is closely associated with other poor risk features, especially $\mathrm{t}(4 ; 14)$, which harbour del(13q) in $80 \%$ of cases $(28-30)$.

\section{One or more lesions?}

Data from the MRC Myeloma IX trial suggested adverse IgH translocation [i.e. $\mathrm{t}(4 ; 14), \mathrm{t}(14 ; 16)$, $\mathrm{t}(14 ; 20)], 1 \mathrm{q}+$ and $\operatorname{del}(17 \mathrm{p})$ each had a similar negative impact on survival when present individually. There was an association between the number of adverse lesions present and progressively shorter survival (OS if no adverse lesions 60.6 months, 1 adverse lesion 41.9 months, $>1$ adverse lesion 21.7 months) (28).

\section{FISH abnormalities at relapse}

The majority of studies reporting the impact of cytogenetics on survival in myeloma have investigated newly diagnosed patients. Evidence also exists for the prognostic impact of cytogenetics at relapse. For example, $\mathrm{t}(4 ; 14)$ is associated with aggressive disease at relapse (54), including resistance to alkylating agents at relapse post ASCT and only short duration of response to thalidomide (55). A single centre study of FISH analysis at relapse in 154 consecutive patients found that patients with adverse cytogenetic abnormalities detected at relapse $[\mathrm{t}(4 ; 14), \mathrm{t}(14 ; 16)$, del(17p), del(1p), 1q+] had significantly inferior PFS (5.6 months vs 9.8) and OS (15.6 months vs not reached) compared to those with standard risk FISH [t(11;14), normal or other] (51). Proteasome inhibitor based therapy at relapse significantly improved survival for high risk patients, achieving similar survival outcomes to those with standard risk FISH.

\section{Recurrent mutations}

Activating mutations are found in a number of genes in myeloma, with frequencies varying in different studies e.g. NRAS (20-30\%), KRAS (15-20\%), BRAF (5\%), CCND1 (12\%), MYC (1\%) and a number of genes in the NF-кB pathway (6-17\%) (27, 50, 56). Mutations in TP53 are present in around one third of cases with del(17p) but are much less prevalent in patients without del(17p). Mutations in other genes in the DNA repair/apoptosis pathway (e.g. ATM, 3\% and ATR, 1.5\%) are also seen (50).

There are conflicting reports regarding the prognostic significance of NRAS and KRAS mutations. Studies performed prior to the novel agent era reported the poor prognostic impact of $K R A S$ mutations (56). Recently, results from the APEX trial suggested that NRAS mutations conferred 
resistance to single agent bortezomib in previously treated patients (57). In 463 newly diagnosed patients treated intensively and non-intensively with novel therapies in the NCRN Myeloma XI trial, the RAS/MAPK pathway was the most frequently mutated pathway but was prognostically neutral (50). In the same study, CCND1 mutations were associated with $\mathrm{t}(11 ; 14)$ and had a negative impact on OS ( 2 year OS $38.1 \%$ vs $80 \%$ ). Mutations preventing apoptotic signalling also had a negative impact on outcome, and when combined, ATM, ATR and TP53 mutations and del(17p) had a significant impact on both PFS and OS (50). In a multivariate analysis for OS, TP53, CCND1, ATM and ATR mutations were independent prognostic factors. In contrast, mutations in IRF4 (mutated in 3.2\% cases) and EGR1 (3.7\%), both of which are IMiD target genes, were associated with a favourable OS. When poor prognostic mutations were combined with adverse cytogenetic abnormalities, a cumulative negative impact was seen. Patients with 2 or more adverse lesions accounted for $13 \%$ of the patient group and had a 2 year OS $45 \%$ vs $83 \%$ for patients with less than 2 adverse lesions.

The existence of intra-clonal heterogeneity in myeloma tumours has become increasingly apparent (58). In addition to mutational load being a poor prognostic factor, analysis of patients from the same Myeloma XI trial showed that increased subclonal diversity was associated with poor outcome (59).

\section{EXPRESSION AND EPIGENETIC SIGNATURES}

Several groups have used gene expression profiling (GEP) to risk stratify myeloma patients. The first model was described by the Arkansas group (32). A 70-gene high risk signature, seen in $13 \%$ of patients treated on Total Therapy 2 (TT2) and Total Therapy 3 (TT3), was associated with inferior event free survival (5 year EFS $18 \%$ vs $60 \%$ p $<0.01$ ) and OS (5 year OS $28 \%$ vs $78 \%$ $\mathrm{p}<0.01)$. Further analysis reduced these genes down to 17 , with over-representation of upregulated genes from chromosome 1q, and downregulated genes mapping to 1p. Analysis of sequential samples taken at diagnosis and subsequent relapse showed the percentage of patients with a high risk score increased at relapse, from $13 \%$ to $76 \%$ and this was again associated with shorter OS. The same group derived an expression signature, GEP80, correlating with resistance to bortezomib and found enrichment for genes involved in protein ubiquitination pathways (60). One of these, PSMD4, resides on chromosome 1q and its expression is sensitive to copy number. In TT3, PFS and OS progressively shortened with increased copy number of chromosome 1q and PSMD4 expression. This is a potential explanation for the data from the HOVON-65/GMMG-HD4 study, discussed above, where half of the patients were treated with bortezomib, and overall inferior outcomes were seen with increasing 1q copy number (47).

The IFM 15 gene model based on newly diagnosed patients treated on the IFM-99 clinical trials, predicted 3-year survival of $90.5 \%$ in the low risk groups vs $47.4 \%$ in the high risk group, and was validated in some other clinical cohorts (61). The high risk group was characterised by overexpression of genes involved in mitosis and when cytogenetics were analysed, this group was associated with del(17p), 1q+ and $\mathrm{t}(4 ; 14)$ FISH abnormalities whilst the low risk group was associated with hyperdiploidy. Combining high risk IFM-15 model with presence of $\mathrm{t}(4 ; 14)$ and Beta 2 microglobulin $\geq 5.5$ produced a high risk group with 3 year OS of only $34 \%$.

The EMC-92 GEP signature was developed using the HOVON-65/GMMG-HD4 data set then subsequently validated on data sets from TT2 (thalidomide based), TT3 (bortezmib based), MRCIX (transplant eligible and non-eligible) and APEX (relapsed patients) clinical trials (62) . In each case a high risk population was defined, accounting for $16-24 \%$ of the patient population. Thus, this was shown to be highly discriminating irrespective of treatment regimen, age and relapse 
status. Again, enrichment of the long arm of chromosome 1 was seen in the probe sets used in this study. When cytogenetic abnormalities within the EMC-92 and UAMS-70 signatures were analysed, poor prognostic cytogenetic abnormalities [defined as $1 \mathrm{q}+, \operatorname{del}(17 \mathrm{p}), \mathrm{t}(4 ; 14), \mathrm{t}(14 ; 16)$, $t(14 ; 20)$ and del(13q)] were enriched in the high risk groups.

\section{DNA Methylation}

The prognostic significance of epigenetic regulators has also been explored by examining the association between methylation status and prognosis in myeloma. Both hypo- and hypermethylation of various genes has been implicated in prognosis. Kaiser et al (63) analysed samples from both intensive and non-intensive arms of MRC-IX trial and found that hypermethylation of four tumour suppressor genes $(G P X 3, R B P 1, S P A R C$ and $T G F B I)$ was associated with decreased gene expression and significantly shorter OS, independent of age, ISS score and cytogenetics. Methylation status was able to distinguish two subgroups within the hyperdiploid group with differing outcomes (64). Global hypomethylation has been shown to be associated with a poorer outcome in myeloma patients (OS 11.9 vs 61.7 months) (65).

\section{microRNAs}

MicroRNAs are small, non-coding, single stranded RNAs, typically 19-25 nucleotides long, which regulate gene expression by binding to complementary sites in mRNA, resulting in mRNA degradation and inhibition of protein translation. They have been shown to regulate critical genes involved in the pathogenesis of myeloma, including the TP53/MDM2 (66) pathway and are variously up or down regulated in myeloma. Different genetic subgroups have distinct patterns of microRNA expression (67). Expression levels of certain microRNAs have been shown to be associated with OS $(66,67)$.

\section{RADIOLOGICAL FEATURES AND EFFECT OF THERAPY}

Imaging at diagnosis has been shown to be associated with prognosis. In TT3, numbers of focal lesions detected by MRI and PET/CET, and metabolic activity, were positively linked to GEP high risk disease (68). Eight or more focal lesions on MRI of the axial skeleton was associated with inferior EFS, but not OS, as was the degree of uptake on PET/CT. On further analysis, the prognostic impact of FDG-avid CT/PET lesions was confined to gene-array defined low risk patients. Patients with high risk myeloma, defined by GEP, all fared badly, irrespective of CT/PET findings (69). Similar results were obtained in an Italian study in which patients received thalidomide/dexamethasone induction followed by tandem ASCT. (18). Presence of at least 3 focal lesions, degree of uptake and extramedullary disease were all associated with shorter PFS, and the latter two feature were also associated with shorter OS. Newly diagnosed patients with a diffuse pattern of marrow infiltration on MRI are reported to have inferior survival compared to those with other patterns (focal, variegated and normal), although this was partly overcome by the use of novel agent-based therapies(70).

Changes in MRI defined focal lesions post-treatment have been reported to correlate with response to therapy. The number of focal lesions present on a post-ASCT MRI scan had prognostic significance for both PFS and OS (71). In TT2 trial, resolution of focal lesions on axial MRI was associated with superior survival (72), suggesting residual focal lesions may be a source of relapse. One potential disadvantage of MRI is the concern it provides false positives due to nonviable lesions (73). In TT3 trial, complete FDG suppression in previously avid PET/CT lesions post induction therapy correlated with superior EFS and OS (69). In a retrospective study of 191 
patients, imaging changes occurred faster on PET/CT than MRI in patients who responded to therapy (73). In keeping with this, $>3$ PET positive lesions on day 7 of induction in the TT3 trial were associated with a poorer outcome. The prognostic significance of the 7 day PET/CT remained in the GEP high risk sub-group (74). The Italian study discussed above also found that persistent FDG positive lesions after thalidomide/dexamethasone induction was associated with shorter PFS whilst PET negativity 3 months post ASCT predicted improved PFS and OS (18). The IFM/DFCI 2009 trial (bortezomib, lenalidomide and dexamethasone induction) compared PET/CT with MRI and preliminary results suggest normalisation of PET/CT is the superior predictor for survival (75).

\section{STAGING SYSTEMS}

\section{International Staging System}

The International Staging System (ISS) replaced the previous Durie-Salmon staging system and defines 3 categories (Table 2). It reflects tumour burden and renal function (Beta-2 microglobulin) along with general performance status (albumin). The ISS retains prognostic value for conventional chemotherapy, high dose therapy and novel agents $(76,77)$, and is also prognostic for overall survival at relapse (78).

\section{Combined ISS and Cytogenetics}

Several groups have reported that combining ISS score with cytogenetic risk can more accurately predict prognosis than using either tool alone. Boyd et al analysed 1960 patients treated on the MRC Myeloma IX trials. They combined ISS stage with the presence of 0,1 or $>1$ adverse genetic lesions (adverse IGH translocation, $\operatorname{del}(17 \mathrm{p})$ or $1 \mathrm{q}+$ ) to define 3 risk categories. Median OS in the lowest risk group (ISS I or II with no adverse lesion or ISS I with 1 adverse lesion) was 67.8 months versus 19.4 months in the highest risk group (ISS II or III plus $>1$ adverse lesion), which accounted for $13.8 \%$ of patients (28). An International Myeloma Working Group (IMWG) collaborative project retrospectively analysed 2642 patients treated worldwide, with $59 \%$ treated intensively. ISS stage combined with the presence or absence of $\mathrm{t}(4 ; 14)$ and/or del(17p) created 3 risk groups. The lowest risk group had 4 year median OS of $71 \%$ vs $33 \%$ for the highest risk group (79). This was confirmed by Neben et al, who showed that the same combined ISS score with $\mathrm{t}(4 ; 14)$ and del(17p) again predicted PFS and OS much better than ISS alone, specifically in patients undergoing autologous stem cell transplant (80).

\section{Revised-International Staging System}

Recently the IMWG has taken the concept of combining ISS with genetic risk factors a stage further to produce the Revised International Staging System (R-ISS) (81). Data from 4445 patients with newly diagnosed myeloma enrolled onto 11 international trials were pooled. In all but one trial, patients received an IMiD or proteasome inhibitor as part of their therapy. Patients were risk stratified according to ISS stage, presence or absence of high risk cytogenetics, defined as $t(4 ; 14)$, $\mathrm{t}(14 ; 16)$ and $\operatorname{del}(17 \mathrm{p})$ and normal or high serum LDH. Combining these parameters, three risk groups were determined, as outlined in Table 3.

\section{QUALITY AND DEPTH OF RESPONSE}

A number of studies have established the link between depth of response and improved OS (82, 83). The IFM group showed attaining at least Very Good Partial Response (VGPR) was associated with a favourable outcome (83) in patients treated with bortezomib and dexamethasone or VAD. 
Patients who achieved $\geq$ VGPR post induction had longer PFS compared with those who only achieved $\geq$ VGPR following high dose therapy plus ASCT (41.2 months vs 31.1, $\mathrm{p}=0.01$ ).

A retrospective analysis of newly diagnosed elderly patients treated with novel agents showed 3 year OS was 91\%, 70\% and 67\% for patients achieving Complete Response (CR), VGPR and Partial Response (PR) respectively (82). Analysis of 344 patients undergoing ASCT in Spain showed patients achieving CR had significantly longer OS compared to those achieving nCR (35\% vs $22 \%$ OS at 12 years), with no significant difference in OS between patients achieving nCR, VGPR or PR (84).

A single centre analysis of autologous stem cell transplant outcomes in 338 consecutive patients, found that early relapse post ASCT was the most important predictor of OS (median OS 1.6 years if relapse within 12 months vs 7.2 years if not) and appeared to outweigh the impact of high risk cytogenetics (85), with other studies showing similar results (86).

The importance of achieving a CR for long term outcome may not apply to all groups. In TT2, CR was predictive in the high risk GEP group only (87). In the same cohort, achieving CR had no significant impact on outcome in patients with a history of prior MGUS or asymptomatic myeloma (88). Symptomatic myeloma patients with an MGUS-like gene expression profile had lower CR rates but superior survival (89).

Stringent CR criteria (sCR) was introduced by IMWG in 2006, adding normal SFLC ratio plus the absence of clonal plasma cells in the marrow to the pre-existing CR criteria. Only one study has reported benefit of sCR over CR however, whilst others have suggested SFLC values do not provide additional prognostic information (90).

\section{Minimal Residual Disease (MRD)}

There are three main methods to detect MRD in myeloma: next generation sequencing (NGS) of immunoglobulin genes, MFC and sensitive imaging techniques (e.g. CT-PET), which are important for extramedullary disease/relapse. NGS is highly sensitive, $\left(10^{-6}\right)$ but relatively time consuming and a diagnostic sample is mandatory $(91,92)$. MFC does not necessarily require a diagnostic sample, is less time consuming than NGS and provides intra-assay validation, since haematopoietic precursors can be detected, identifying haemodilute samples (91). Sensitivity is generally lower than that achieved by NGS $\left(10^{-4}\right.$ to $10^{-5}$ using 6 colour flow), although $10^{-5}$ to $10^{-}$ ${ }^{6}$ may be possible with 8 colour flow.

MRD negativity, monitored by MFC, is associated with prolonged PFS and OS, in transplant eligible and ineligible patients $(91,93)$. In the intensive arm of the MRC Myeloma IX trial, median OS was 59.0 months for patients who were MRD positive after ASCT versus 80.6 months in MRD negative patients (93). This outcome advantage was seen in patients with standard and high risk cytogenetics.

MRD analysis by NGS was performed on 133 patients treated in GEM clinical trials, both transplant eligible and ineligible, some of whom were treated with novel agents. Patients who were MRD negative had a significantly longer time to progression and OS (median not reached vs 81 months) compared with MRD positive patients (92).

Considering MRD level as a continuous variable can improve its predictive value. In the MRC Myeloma IX trial intensive arm, approximately 1 year median OS benefit was noted per log 
depletion of MRD level (94). MRD levels assessed by NGS can also be quantitative. MartinezLopez et al found when patients were stratified according to different levels of MRD, TTP medians were as follows: MRD $\geq 10^{-3} 27$ months, MRD $10^{-3}$ to $10^{-5} 48$ months and MRD $<10^{5} 80$ months (92).

Two independent studies have shown that MRD positivity in the bone marrow can identify patients with solitary plasmacytomas at high risk of progression to multiple myeloma. At a median of 26 months follow up, $71 \%$ and $72 \%$ of patients with solitary bone plasmacytoma plus MRD positivity detected by MFC progressed to myeloma compared to $6 \%$ and $12 \%$ of patients with negative bone marrow results respectively (95).

MRD assessment has prognostic value in relapsed myeloma. Patients in the BSBMT/UKMF Myeloma X trial were treated with PAD re-induction followed by randomisation to consolidation with second ASCT or weekly cyclophosphamide. MRD analysis by MFC post-consolidation in patients achieving CR revealed longer OS in those who were MRD negative (25 months vs 10 months) (96).

\section{Predictive Biomarkers}

Prognostic markers provide information about outcome, whereas predictive markers provide specific information about the likelihood of response to particular drugs or regimens. Prognostic markers are important for risk stratification, which may influence therapeutic choices, whereas predictive markers are useful for individualising treatment. A marker can be prognostic, predictive or both. Until recently, few if any predictive markers were known in myeloma. More recently, it has been shown that cereblon expression is required for the anti-myeloma activity of lenalidomide and pomalidomide (97). In a study of 53 refractory myeloma patients treated with pomalidomide and dexamethasone, no patient with very low cereblon expression responded. Cereblon levels predicted for significant differences in PFS (3.0 months vs 8.9, lowest quartile vs highest three quartiles) and OS (9.1 months vs 27.2) (98).

The presence of RAS mutations, or mutations in the RAS/MAPK pathway, may identify a group of patients suitable for treatment with MEK inhibitors (99), whilst BRAF mutations may be amenable to BRAF inhibition (58). Mutations in the NF-KB pathway could also be targeted therapeutically (50).

\section{Integrating risk factors and staging systems for practical use}

The R-ISS is an attempt at integrating both host and tumour-related factors to produce an estimate of survival, however it is clear that other factors also contribute to the risk for each patient. Table 4 summarises the most significant factors, including the quality of response and speed of relapse. The R-ISS is a useful tool to guide patient counselling, and choice of regimen in newly diagnosed patients requiring treatment. For example, patients with Stage 3 disease should be treated with a proteasome inhibitor and IMiD combination, where available and depending on patient fitness. Choice of first line regimen will increasingly be influenced by the baseline risk group that a patient falls into. While evidence from prospective studies is lacking, accumulating data from sub-group analyses of large phase 3 studies provide the basis for current guidance (100). Adverse disease features at baseline not included in the R-ISS such as extra-medullary disease may be used to "up-stage" a patient, in whom attempts should be made to achieve a functional response (PET-CT negative) following initial therapy. 
Patients who relapse early following completion of frontline therapy clearly have high risk disease and should be entered into clinical trials where available, and the acquisition of additional genetic lesions at relapse may also alter risk, as indicated in a recent retrospective study (51).

\section{$\underline{\text { Summary }}$}

Our understanding of the factors that influence prognosis in myeloma has advanced considerably so that we now recognise the contribution of a range of features that include patient fitness, disease biology, genetic lesions, radiological features and depth of response, including MRD analysis. Prognostic factors can be combined to give greater information, as in the R-ISS. Understanding prognosis in myeloma will remain an evolving concept for the foreseeable future due to the development and increasing accessibility of new tools, e.g. GEP, MRD analysis, as well as the need to revalidate pre-existing models as therapies advance. One of the key challenges will be to establish links between prognostic factors and the relative importance of different factors.

Until recently, prognostic information in myeloma was mainly used for patient information and managing expectations. With an increasing choice of ever improving regimens with varying intensities and toxicity we may now be able to use risk stratification to adapt treatment strategy, both at diagnosis, and further along the treatment pathway, where the use of MRD tools may identify some patients for intensification, and others to de-escalation of therapy. Risk adapted strategies have yet to be assessed in prospective clinical trials, an issue which is likely to be addressed with current trial designs. Patient choice and priorities will increasingly feature in treatment decisions, and prospective studies should include quality of life assessments in order to better inform patients and clinicians undertaking such decisions.

Such risk adapted approaches would signify the first step towards individualised therapy. Further advances towards this have been achieved by the recent discovery of predictive biomarkers in myeloma. Pending validation of these predictive biomarkers in prospective clinical trials, integration with multi-modality prognostic information should lead to a future myeloma treatment landscape which is personalized and more efficacious.

Contributors: DS and KY designed the article, drafted and revised it and approved the final manuscript. 
1. Chretien ML, Hebraud B, Cances-Lauwers V, Hulin C, Marit G, Leleu X, et al. Age is a prognostic factor even among patients with multiple myeloma younger than 66 years treated with highdose melphalan: the IFM experience on 2316 patients. Haematologica. 2014;99(7):1236-8.

2. Avet-Loiseau H, Hulin C, Campion L, Rodon P, Marit G, Attal M, et al. Chromosomal abnormalities are major prognostic factors in elderly patients with multiple myeloma: the intergroupe francophone du myélome experience. J Clin Oncol. 2013;31(22):2806-9.

3. Auner HW, Garderet L, Kröger N. Autologous haematopoietic cell transplantation in elderly patients with multiple myeloma. Br J Haematol. 2015;171(4):453-62.

4. $\quad$ Garderet L, Touzeau C, Stoppa A-M, Caillot D, Karlin L, Moreau P, et al. Upfront Autologous Stem Cell Translpantation for Newly Diagnosed Elderly Multiple Myeloma (MM) Patients: A Prospective Multicenter Study. ASH; Orlando: Blood; 2015.

5. Palumbo A, Bringhen S, Mateos MV, Larocca A, Facon T, Kumar SK, et al. Geriatric assessment predicts survival and toxicities in elderly myeloma patients: an International Myeloma Working Group report. Blood. 2015;125(13):2068-74.

6. Sigurdardottir EE, Turesson I, Lund SH, Lindqvist EK, Mailankody S, Korde N, et al. The Role of Diagnosis and Clinical Follow-up of Monoclonal Gammopathy of Undetermined Significance on Survival in Multiple Myeloma. JAMA Oncol. 2015;1(2):168-74.

7. $\quad$ Smith D, Yong K. Multiple myeloma. BMJ. 2013;346:f3863.

8. Augustson BM, Begum G, Dunn JA, Barth NJ, Davies F, Morgan G, et al. Early mortality after diagnosis of multiple myeloma: analysis of patients entered onto the United kingdom Medical Research Council trials between 1980 and 2002--Medical Research Council Adult Leukaemia Working Party. J Clin Oncol. 2005;23(36):9219-26.

9. Dimopoulos MA, Terpos E, Chanan-Khan A, Leung N, Ludwig H, Jagannath S, et al. Renal impairment in patients with multiple myeloma: a consensus statement on behalf of the International Myeloma Working Group. J Clin Oncol. 2010;28(33):4976-84.

10. Haynes RJ, Read S, Collins GP, Darby SC, Winearls CG. Presentation and survival of patients with severe acute kidney injury and multiple myeloma: a 20 -year experience from a single centre. Nephrol Dial Transplant. 2010;25(2):419-26.

11. Alexandrakis MG, Passam FH, Kyriakou DS, Dambaki K, Niniraki M, Stathopoulos E. Ki-67 proliferation index: correlation with prognostic parameters and outcome in multiple myeloma. Am J Clin Oncol. 2004;27(1):8-13.

12. Hose D, Rème T, Hielscher T, Moreaux J, Messner T, Seckinger A, et al. Proliferation is a central independent prognostic factor and target for personalized and risk-adapted treatment in multiple myeloma. Haematologica. 2011;96(1):87-95.

13. Greipp PR, Lust JA, O'Fallon WM, Katzmann JA, Witzig TE, Kyle RA. Plasma cell labeling index and beta 2-microglobulin predict survival independent of thymidine kinase and C-reactive protein in multiple myeloma. Blood. 1993;81(12):3382-7.

14. Paiva B, Vídriales MB, Montalbán M, Pérez JJ, Gutiérrez NC, Rosiñol L, et al. Multiparameter flow cytometry evaluation of plasma cell DNA content and proliferation in 595 transplant-eligible patients with myeloma included in the Spanish GEM2000 and GEM2005<65y trials. Am J Pathol. 2012;181(5):1870-8.

15. Moreau P, Facon T, Leleu X, Morineau N, Huyghe P, Harousseau JL, et al. Recurrent 14q32 translocations determine the prognosis of multiple myeloma, especially in patients receiving intensive chemotherapy. Blood. 2002;100(5):1579-83.

16. Pandey S, Kyle RA. Unusual myelomas: a review of $\operatorname{IgD}$ and $\operatorname{IgE}$ variants. Oncology (Williston Park). 2013;27(8):798-803.

17. Usmani SZ, Heuck C, Mitchell A, Szymonifka J, Nair B, Hoering A, et al. Extramedullary disease portends poor prognosis in multiple myeloma and is over-represented in high-risk disease even in the era of novel agents. Haematologica. 2012;97(11):1761-7.

18. Zamagni E, Patriarca F, Nanni C, Zannetti B, Englaro E, Pezzi A, et al. Prognostic relevance of 18-F FDG PET/CT in newly diagnosed multiple myeloma patients treated with up-front autologous transplantation. Blood. 2011;118(23):5989-95. 
19. Rosiñol L, Oriol A, Teruel AI, Hernández D, López-Jiménez J, de la Rubia J, et al. Superiority of bortezomib, thalidomide, and dexamethasone (VTD) as induction pretransplantation therapy in multiple myeloma: a randomized phase 3 PETHEMA/GEM study. Blood. 2012;120(8):1589-96.

20. Fernández de Larrea C, Kyle RA, Durie BG, Ludwig H, Usmani S, Vesole DH, et al. Plasma cell leukemia: consensus statement on diagnostic requirements, response criteria and treatment recommendations by the International Myeloma Working Group. Leukemia. 2013;27(4):780-91.

21. Drake MB, Iacobelli S, van Biezen A, Morris C, Apperley JF, Niederwieser D, et al. Primary plasma cell leukemia and autologous stem cell transplantation. Haematologica. 2010;95(5):804-9.

22. Mahindra A, Kalaycio ME, Vela-Ojeda J, Vesole DH, Zhang MJ, Li P, et al. Hematopoietic cell transplantation for primary plasma cell leukemia: results from the Center for International Blood and Marrow Transplant Research. Leukemia. 2012;26(5):1091-7.

23. Gonsalves WI, Rajkumar SV, Gupta V, Morice WG, Timm MM, Singh PP, et al. Quantification of clonal circulating plasma cells in newly diagnosed multiple myeloma: implications for redefining highrisk myeloma. Leukemia. 2014;28(10):2060-5.

24. Lee J, Smith D, Rabin N, Tobias J, Yong K. 17P deleted multiple myeloma presenting with intracranial disease: durable remission after tailored management. Hematol Oncol. 2015.

25. Terpos E, Katodritou E, Roussou M, Pouli A, Michalis E, Delimpasi S, et al. High serum lactate dehydrogenase adds prognostic value to the international myeloma staging system even in the era of novel agents. Eur J Haematol. 2010;85(2):114-9.

26. Chesi M, Bergsagel PL. Molecular pathogenesis of multiple myeloma: basic and clinical updates. Int J Hematol. 2013;97(3):313-23.

27. Morgan GJ, Walker BA, Davies FE. The genetic architecture of multiple myeloma. Nat Rev Cancer. 2012;12(5):335-48.

28. Boyd KD, Ross FM, Chiecchio L, Dagrada GP, Konn ZJ, Tapper WJ, et al. A novel prognostic model in myeloma based on co-segregating adverse FISH lesions and the ISS: analysis of patients treated in the MRC Myeloma IX trial. Leukemia. 2012;26(2):349-55.

29. Bergsagel PL, Mateos MV, Gutierrez NC, Rajkumar SV, San Miguel JF. Improving overall survival and overcoming adverse prognosis in the treatment of cytogenetically high-risk multiple myeloma. Blood. 2013;121(6):884-92.

30. Avet-Loiseau H, Attal M, Moreau P, Charbonnel C, Garban F, Hulin C, et al. Genetic abnormalities and survival in multiple myeloma: the experience of the Intergroupe Francophone du Myélome. Blood. 2007;109(8):3489-95.

31. Moreau P, Attal M, Garban F, Hulin C, Facon T, Marit G, et al. Heterogeneity of t( $4 ; 14)$ in multiple myeloma. Long-term follow-up of 100 cases treated with tandem transplantation in IFM99 trials. Leukemia. 2007;21(9):2020-4.

32. Shaughnessy JD, Zhan F, Burington BE, Huang Y, Colla S, Hanamura I, et al. A validated gene expression model of high-risk multiple myeloma is defined by deregulated expression of genes mapping to chromosome 1. Blood. 2007;109(6):2276-84.

33. Avet-Loiseau H, Leleu X, Roussel M, Moreau P, Guerin-Charbonnel C, Caillot D, et al. Bortezomib plus dexamethasone induction improves outcome of patients with $\mathrm{t}(4 ; 14)$ myeloma but not outcome of patients with del(17p). J Clin Oncol. 2010;28(30):4630-4.

34. Cavo M, Pantani L, Petrucci MT, Patriarca F, Zamagni E, Donnarumma D, et al. Bortezomibthalidomide-dexamethasone is superior to thalidomide-dexamethasone as consolidation therapy after autologous hematopoietic stem cell transplantation in patients with newly diagnosed multiple myeloma. Blood. 2012;120(1):9-19.

35. Nair B, van Rhee F, Shaughnessy JD, Anaissie E, Szymonifka J, Hoering A, et al. Superior results of Total Therapy 3 (2003-33) in gene expression profiling-defined low-risk multiple myeloma confirmed in subsequent trial 2006-66 with VRD maintenance. Blood. 2010;115(21):4168-73.

36. Mikhael JR, Dingli D, Roy V, Reeder CB, Buadi FK, Hayman SR, et al. Management of newly diagnosed symptomatic multiple myeloma: updated Mayo Stratification of Myeloma and Risk-Adapted Therapy (mSMART) consensus guidelines 2013. Mayo Clin Proc. 2013;88(4):360-76.

37. Fonseca R, Blood E, Rue M, Harrington D, Oken MM, Kyle RA, et al. Clinical and biologic implications of recurrent genomic aberrations in myeloma. Blood. 2003;101(11):4569-75.

38. Avet-Loiseau H, Malard F, Campion L, Magrangeas F, Sebban C, Lioure B, et al. Translocation $\mathrm{t}(14 ; 16)$ and multiple myeloma: is it really an independent prognostic factor? Blood. 2011;117(6):200911. 
39. Ross FM, Chiecchio L, Dagrada G, Protheroe RK, Stockley DM, Harrison CJ, et al. The t(14;20) is a poor prognostic factor in myeloma but is associated with long-term stable disease in monoclonal gammopathies of undetermined significance. Haematologica. 2010;95(7):1221-5.

40. Bochtler T, Hegenbart U, Kunz C, Granzow M, Benner A, Seckinger A, et al. Translocation $\mathrm{t}(11 ; 14)$ is associated with adverse outcome in patients with newly diagnosed AL amyloidosis when treated with bortezomib-based regimens. J Clin Oncol. 2015;33(12):1371-8.

41. Sasaki K, Lu G, Saliba RM, Bashir Q, Hosing C, Popat U, et al. Impact of $t(11 ; 14)(q 13 ; q 32)$ on the outcome of autologous hematopoietic cell transplantation in multiple myeloma. Biol Blood Marrow Transplant. 2013;19(8):1227-32.

42. Walker BA, Wardell CP, Brioli A, Boyle E, Kaiser MF, Begum DB, et al. Translocations at 8q24 juxtapose MYC with genes that harbor superenhancers resulting in overexpression and poor prognosis in myeloma patients. Blood Cancer J. 2014;4:e191.

43. Smadja NV, Bastard C, Brigaudeau C, Leroux D, Fruchart C, Hématologique GFdC. Hypodiploidy is a major prognostic factor in multiple myeloma. Blood. 2001;98(7):2229-38.

44. Kumar S, Fonseca R, Ketterling RP, Dispenzieri A, Lacy MQ, Gertz MA, et al. Trisomies in multiple myeloma: impact on survival in patients with high-risk cytogenetics. Blood. 2012;119(9):21005.

45. Pawlyn C, Melchor L, Murison A, Wardell CP, Brioli A, Boyle EM, et al. Coexistent hyperdiploidy does not abrogate poor prognosis in myeloma with adverse cytogenetics and may precede IGH translocations. Blood. 2015;125(5):831-40.

46. Hanamura I, Stewart JP, Huang Y, Zhan F, Santra M, Sawyer JR, et al. Frequent gain of chromosome band 1q21 in plasma-cell dyscrasias detected by fluorescence in situ hybridization: incidence increases from MGUS to relapsed myeloma and is related to prognosis and disease progression following tandem stem-cell transplantation. Blood. 2006;108(5):1724-32.

47. Neben K, Lokhorst HM, Jauch A, Bertsch U, Hielscher T, van der Holt B, et al. Administration of bortezomib before and after autologous stem cell transplantation improves outcome in multiple myeloma patients with deletion 17p. Blood. 2012;119(4):940-8.

48. Caltagirone S, Ruggeri M, Aschero S, Gilestro M, Oddolo D, Gay F, et al. Chromosome 1 abnormalities in elderly patients with newly diagnosed multiple myeloma treated with novel therapies. Haematologica. 2014;99(10):1611-7.

49. Fonseca R, Van Wier SA, Chng WJ, Ketterling R, Lacy MQ, Dispenzieri A, et al. Prognostic value of chromosome 1q21 gain by fluorescent in situ hybridization and increase CKS1B expression in myeloma. Leukemia. 2006;20(11):2034-40.

50. Walker BA, Boyle EM, Wardell CP, Murison A, Begum DB, Dahir NM, et al. Mutational Spectrum, Copy Number Changes, and Outcome: Results of a Sequencing Study of Patients With Newly Diagnosed Myeloma. J Clin Oncol. 2015;33(33):3911-20.

51. Smith D, Stephenson C, Percy L, Lach A, Chatters S, Kempski H, et al. Cohort analysis of FISH testing of CD138(+) cells in relapsed multiple myeloma: implications for prognosis and choice of therapy. Br J Haematol. 2015.

52. Boyd KD, Ross FM, Walker BA, Wardell CP, Tapper WJ, Chiecchio L, et al. Mapping of chromosome $1 \mathrm{p}$ deletions in myeloma identifies FAM46C at $1 \mathrm{p} 12$ and CDKN2C at $1 \mathrm{p} 32.3$ as being genes in regions associated with adverse survival. Clin Cancer Res. 2011;17(24):7776-84.

53. Ross FM, Avet-Loiseau H, Ameye G, Gutiérrez NC, Liebisch P, O'Connor S, et al. Report from the European Myeloma Network on interphase FISH in multiple myeloma and related disorders. Haematologica. 2012;97(8):1272-7.

54. Karlin L, Soulier J, Chandesris O, Choquet S, Belhadj K, Macro M, et al. Clinical and biological features of $\mathrm{t}(4 ; 14)$ multiple myeloma: a prospective study. Leuk Lymphoma. 2011;52(2):238-46.

55. Jaksic W, Trudel S, Chang H, Trieu Y, Qi X, Mikhael J, et al. Clinical outcomes in t $4 ; 14$ ) multiple myeloma: a chemotherapy-sensitive disease characterized by rapid relapse and alkylating agent resistance. J Clin Oncol. 2005;23(28):7069-73.

56. Smith D, Armenteros E, Percy L, Kumar M, Lach A, Herledan G, et al. RAS mutation status and bortezomib therapy for relapsed multiple myeloma. Br J Haematol. 2015;169(6):905-8.

57. Mulligan G, Lichter DI, Di Bacco A, Blakemore SJ, Berger A, Koenig E, et al. Mutation of NRAS but not KRAS significantly reduces myeloma sensitivity to single-agent bortezomib therapy. Blood. 2014;123(5):632-9. 
58. Brioli A, Melchor L, Cavo M, Morgan GJ. The impact of intra-clonal heterogeneity on the treatment of multiple myeloma. Br J Haematol. 2014;165(4):441-54.

59. Melchor L, Murison A, Boyle E, Wardell C, Jones J, Kaiser M, et al. The Extent of Intra-Clonal Genetic Diversity within the Myeloma Clone Is a Predictive Biomarker of Progression and Outcome after Treatment. ASH; San Francisco: Blood; 2014.

60. Shaughnessy JD, Qu P, Usmani S, Heuck CJ, Zhang Q, Zhou Y, et al. Pharmacogenomics of bortezomib test-dosing identifies hyperexpression of proteasome genes, especially PSMD4, as novel high-risk feature in myeloma treated with Total Therapy 3. Blood. 2011;118(13):3512-24.

61. Decaux O, Lodé L, Magrangeas F, Charbonnel C, Gouraud W, Jézéquel P, et al. Prediction of survival in multiple myeloma based on gene expression profiles reveals cell cycle and chromosomal instability signatures in high-risk patients and hyperdiploid signatures in low-risk patients: a study of the Intergroupe Francophone du Myélome. J Clin Oncol. 2008;26(29):4798-805.

62. Kuiper R, Broyl A, de Knegt Y, van Vliet MH, van Beers EH, van der Holt B, et al. A gene expression signature for high-risk multiple myeloma. Leukemia. 2012;26(11):2406-13.

63. Kaiser MF, Johnson DC, Wu P, Walker BA, Brioli A, Mirabella F, et al. Global methylation analysis identifies prognostically important epigenetically inactivated tumor suppressor genes in multiple myeloma. Blood. 2013;122(2):219-26.

64. Walker BA, Wardell CP, Chiecchio L, Smith EM, Boyd KD, Neri A, et al. Aberrant global methylation patterns affect the molecular pathogenesis and prognosis of multiple myeloma. Blood. 2011;117(2):553-62.

65. Sive JI, Feber A, Smith D, Quinn J, Beck S, Yong K. Global hypomethylation in myeloma is associated with poor prognosis. Br J Haematol. 2015.

66. Corthals SL, Sun SM, Kuiper R, de Knegt Y, Broyl A, van der Holt B, et al. MicroRNA signatures characterize multiple myeloma patients. Leukemia. 2011;25(11):1784-9.

67. Chi J, Ballabio E, Chen XH, Kušec R, Taylor S, Hay D, et al. MicroRNA expression in multiple myeloma is associated with genetic subtype, isotype and survival. Biol Direct. 2011;6:23.

68. Waheed S, Mitchell A, Usmani S, Epstein J, Yaccoby S, Nair B, et al. Standard and novel imaging methods for multiple myeloma: correlates with prognostic laboratory variables including gene expression profiling data. Haematologica. 2013;98(1):71-8.

69. Bartel TB, Haessler J, Brown TL, Shaughnessy JD, van Rhee F, Anaissie E, et al. F18fluorodeoxyglucose positron emission tomography in the context of other imaging techniques and prognostic factors in multiple myeloma. Blood. 2009;114(10):2068-76.

70. Moulopoulos LA, Dimopoulos MA, Kastritis E, Christoulas D, Gkotzamanidou M, Roussou M, et al. Diffuse pattern of bone marrow involvement on magnetic resonance imaging is associated with high risk cytogenetics and poor outcome in newly diagnosed, symptomatic patients with multiple myeloma: a single center experience on 228 patients. Am J Hematol. 2012;87(9):861-4.

71. Hillengass J, Ayyaz S, Kilk K, Weber MA, Hielscher T, Shah R, et al. Changes in magnetic resonance imaging before and after autologous stem cell transplantation correlate with response and survival in multiple myeloma. Haematologica. 2012;97(11):1757-60.

72. Walker R, Barlogie B, Haessler J, Tricot G, Anaissie E, Shaughnessy JD, et al. Magnetic resonance imaging in multiple myeloma: diagnostic and clinical implications. J Clin Oncol. 2007;25(9):1121-8.

73. Dimopoulos MA, Hillengass J, Usmani S, Zamagni E, Lentzsch S, Davies FE, et al. Role of magnetic resonance imaging in the management of patients with multiple myeloma: a consensus statement. J Clin Oncol. 2015;33(6):657-64.

74. Usmani SZ, Mitchell A, Waheed S, Crowley J, Hoering A, Petty N, et al. Prognostic implications of serial 18-fluoro-deoxyglucose emission tomography in multiple myeloma treated with total therapy 3 . Blood. 2013;121(10):1819-23.

75. Moreau P, Attal M, Karlin L, Garderet L, Facon T, Macro M, et al. Prospective Evaluation of MRI and PET-CT at Diagnosis and before Maintenance Therapy in Symptomatic Patients with Multiple Myeloma Included in the IFM/DFCI 2009 Trial. ASH; Orlando: Blood; 2015.

76. Munshi NC, Anderson KC, Bergsagel PL, Shaughnessy J, Palumbo A, Durie B, et al. Consensus recommendations for risk stratification in multiple myeloma: report of the International Myeloma Workshop Consensus Panel 2. Blood. 2011;117(18):4696-700.

77. Kastritis E, Zervas K, Symeonidis A, Terpos E, Delimbassi S, Anagnostopoulos N, et al. Improved survival of patients with multiple myeloma after the introduction of novel agents and the 
applicability of the International Staging System (ISS): an analysis of the Greek Myeloma Study Group (GMSG). Leukemia. 2009;23(6):1152-7.

78. Kumar SK, Lee JH, Lahuerta JJ, Morgan G, Richardson PG, Crowley J, et al. Risk of progression and survival in multiple myeloma relapsing after therapy with IMiDs and bortezomib: a multicenter international myeloma working group study. Leukemia. 2012;26(1):149-57.

79. Avet-Loiseau H, Durie BG, Cavo M, Attal M, Gutierrez N, Haessler J, et al. Combining fluorescent in situ hybridization data with ISS staging improves risk assessment in myeloma: an International Myeloma Working Group collaborative project. Leukemia. 2013;27(3):711-7.

80. Neben K, Jauch A, Bertsch U, Heiss C, Hielscher T, Seckinger A, et al. Combining information regarding chromosomal aberrations $\mathrm{t}(4 ; 14)$ and del(17p13) with the International Staging System classification allows stratification of myeloma patients undergoing autologous stem cell transplantation. Haematologica. 2010;95(7):1150-7.

81. Palumbo A, Avet-Loiseau H, Oliva S, Lokhorst HM, Goldschmidt H, Rosinol L, et al. Revised International Staging System for Multiple Myeloma: A Report From International Myeloma Working Group. J Clin Oncol. 2015;33(26):2863-9.

82. Gay F, Larocca A, Wijermans P, Cavallo F, Rossi D, Schaafsma R, et al. Complete response correlates with long-term progression-free and overall survival in elderly myeloma treated with novel agents: analysis of 1175 patients. Blood. 2011;117(11):3025-31.

83. Moreau P, Attal M, Pégourié B, Planche L, Hulin C, Facon T, et al. Achievement of VGPR to induction therapy is an important prognostic factor for longer PFS in the IFM 2005-01 trial. Blood. 2011;117(11):3041-4.

84. Martinez-Lopez J, Blade J, Mateos MV, Grande C, Alegre A, García-Laraña J, et al. Long-term prognostic significance of response in multiple myeloma after stem cell transplantation. Blood. 2011;118(3):529-34.

85. Maciocia P, Counsell N, Bird A, Percy L, Moore S, Rabin N, et al. Single Centre Analysis Of Autologous Stem Cell Transplant Outcomes In Multiple Myeloma In 338 Consecutive Patients: Maintenance/Consolidation Is Associated With Superior Survival, But Early Relapse Is The Single Most Important Predictor Of Survival and May Override Genetic Risk. ASH; New Orleans: Blood; 2013. p. 2159-.

86. Kumar S, Mahmood ST, Lacy MQ, Dispenzieri A, Hayman SR, Buadi FK, et al. Impact of early relapse after auto-SCT for multiple myeloma. Bone Marrow Transplant. 2008;42(6):413-20.

87. Haessler J, Shaughnessy JD, Zhan F, Crowley J, Epstein J, van Rhee F, et al. Benefit of complete response in multiple myeloma limited to high-risk subgroup identified by gene expression profiling. Clin Cancer Res. 2007;13(23):7073-9.

88. Pineda-Roman M, Bolejack V, Arzoumanian V, Anaissie E, van Rhee F, Zangari M, et al. Complete response in myeloma extends survival without, but not with history of prior monoclonal gammopathy of undetermined significance or smouldering disease. Br J Haematol. 2007;136(3):393-9.

89. Zhan F, Barlogie B, Arzoumanian V, Huang Y, Williams DR, Hollmig K, et al. Gene-expression signature of benign monoclonal gammopathy evident in multiple myeloma is linked to good prognosis.

Blood. 2007;109(4):1692-700.

90. Martínez-López J, Paiva B, López-Anglada L, Mateos MV, Cedena T, Vidríales MB, et al. Critical analysis of the stringent complete response in multiple myeloma: contribution of sFLC and bone marrow clonality. Blood. 2015;126(7):858-62.

91. Paiva B, van Dongen JJ, Orfao A. New criteria for response assessment: role of minimal residual disease in multiple myeloma. Blood. 2015;125(20):3059-68.

92. Martinez-Lopez J, Lahuerta JJ, Pepin F, González M, Barrio S, Ayala R, et al. Prognostic value of deep sequencing method for minimal residual disease detection in multiple myeloma. Blood. 2014;123(20):3073-9.

93. Rawstron AC, Child JA, de Tute RM, Davies FE, Gregory WM, Bell SE, et al. Minimal residual disease assessed by multiparameter flow cytometry in multiple myeloma: impact on outcome in the Medical Research Council Myeloma IX Study. J Clin Oncol. 2013;31(20):2540-7.

94. Rawstron AC, Gregory WM, de Tute RM, Davies FE, Bell SE, Drayson MT, et al. Minimal residual disease in myeloma by flow cytometry: independent prediction of survival benefit per log reduction. Blood. 2015;125(12):1932-5.

95. Dimopoulos MA, Terpos E. Solitary bone plasmacytomas need to flow. Blood. 2014;124(8):1209-10. 
96. Ashcroft J, de Tute R, Cairns D, Fletcher M, Owen R, Rawstron A, et al. The Utility of Minimal Residual Disease (MRD) Assessment At First Relapse: Results From The BSBMT/Ukmf Myeloma X (Intensive) Trial. ASH; New Orleans: Blood; 2013. p. 3378-.

97. Zhu YX, Braggio E, Shi CX, Bruins LA, Schmidt JE, Van Wier S, et al. Cereblon expression is required for the antimyeloma activity of lenalidomide and pomalidomide. Blood. 2011;118(18):4771-9. 98. Schuster SR, Kortuem KM, Zhu YX, Braggio E, Shi CX, Bruins LA, et al. The clinical significance of cereblon expression in multiple myeloma. Leuk Res. 2014;38(1):23-8.

99. Heuck CJ, Jethava Y, Khan R, van Rhee F, Zangari M, Chavan S, et al. Inhibiting MEK in MAPK pathway-activated myeloma. Leukemia. 2015.

100. Usmani SZ, Rodriguez-Otero P, Bhutani M, Mateos MV, Miguel JS. Defining and treating highrisk multiple myeloma. Leukemia. 2015;29(11):2119-25. 


\begin{tabular}{|l|l|l|l|}
\hline \multicolumn{2}{|c|}{ STANDARD RISK } & \multicolumn{2}{c|}{ HIGH RISK } \\
\hline $\begin{array}{c}\text { CYTOGENETIC } \\
\text { ABNORMALITY }\end{array}$ & \multicolumn{1}{|c|}{ INCIDENCE (\%) } & \multicolumn{1}{c|}{$\begin{array}{c}\text { CYTOGENETIC } \\
\text { ABNORMLAITY }\end{array}$} & INCIDENCE (\%) \\
$\mathrm{t}(11 ; 14)$ & $15 \%$ & $\mathrm{t}(4 ; 14)$ & 15 \\
$\mathrm{t}(6 ; 14)$ & $5 \%$ & $\mathrm{t}(14 ; 16)$ & $2-3$ \\
HYPERDIPLOIDY & $50 \%$ & $\mathrm{t}(14 ; 20)$ & 1 \\
& & $\operatorname{del}(17 \mathrm{p})$ & 10 \\
& & del(1p) & $35-40$ \\
\hline
\end{tabular}

Table 1. Prognostic cytogenetic abnormalities in newly diagnosed multiple myeloma (26, 27).

\begin{tabular}{|c|c|c|}
\hline STAGE I & STAGE II & STAGE III \\
\hline Beta-2 microglobulin $<$ & Not fitting & Beta-2 microglobulin $\geq 5.5 \mathrm{mg} / 1$ \\
$3.5 \mathrm{mg} / 1$ & criteria for stage & \\
AND albumin $\geq 35 \mathrm{~g} / 1$ & I or III & \\
\hline & & \\
\hline
\end{tabular}

Table 2. International Staging System (ISS)

\begin{tabular}{|c|c|c|c|}
\hline & STAGE I & STAGE II & STAGE III \\
\hline & $\begin{array}{c}\text { ISS I } \\
\text { AND standard risk } \\
\text { cytogenetics }{ }^{\mathrm{a}} \text { by FISH } \\
\text { AND normal LDH }\end{array}$ & $\begin{array}{c}\text { Not fitting criteria for } \\
\text { stage I or III }\end{array}$ & $\begin{array}{c}\text { ISS III } \\
\text { AND EITHER high risk } \\
\text { cytogenetics } \text { by FISH }^{\text {OR high LDH }}\end{array}$ \\
\hline $\begin{array}{c}\text { MEDIAN PFS } \\
\text { (months) }\end{array}$ & 66 & 42 & 29 \\
\hline $\begin{array}{c}\text { MEDIAN OS } \\
\text { (months) }\end{array}$ & NOT REACHED & 83 & 43 \\
\hline
\end{tabular}

${ }^{\mathrm{a}}$ Standard risk cytogenetics by FISH = no high risk abnormality

${ }^{\mathrm{b}}$ High risk Cytogenetics by FISH $=\operatorname{del}(17 \mathrm{p})$ and/or $\mathrm{t}(4 ; 14)$ and/or $\mathrm{t}(14 ; 16)$

Table 3. Revised International Staging System (R-ISS) 
Table 4: Summary of prognostic factors

\begin{tabular}{|lll|}
\hline Bumour biology & Baseline & Post-treatment \\
\hline & Genetics* $\S$ & (Further genetic changes)** $^{* *}$ \\
& $\begin{array}{l}\text { Proliferative rate \& Circulating plasma } \\
\text { cells } \\
\text { Extramedullary disease }\end{array}$ & Depth of response \\
& & Timing and tempo of relapse \\
\hline Tumour burden & Beta-2 microglobulin* & Depth of response (CR, \\
& LDH* & Minimal residual disease status) \\
& Age & Treatment emergent toxicity \\
Patient factors & Albumin* & Frailty \\
& Frailty & Age \\
& & \\
\hline
\end{tabular}

* incorporated in R-ISS scoring system

** acquisition of additional genetic lesions such as $\operatorname{del}(17 \mathrm{p})$ or $1 \mathrm{q}$ amplification would indicative of worse prognosis

$\S$ the co-existence of $\geq 2$ adverse features, eg $\mathrm{t}(4 ; 14)$ with $1 \mathrm{q}+$ or with ISS 3 , high LDH or poor response to an optimal induction regimen of a proteasome inhibitor and an IMiD confers an "ultra high" risk associated with a survival of under 2 years (100)

\section{$\underline{\text { ABSTRACT }}$}

Outcomes in multiple myeloma have greatly improved over the last decade due to the introduction of newer, more effective therapies. This improvement is not uniform however and it is increasingly apparent that myeloma is a heterogeneous disease, with some patients living for 1-2 years whilst others are alive and progression free at 10 years. This variation in outcome is due to patient characteristics plus features intrinsic to the myeloma tumour. Determining such prognostic factors is important not only for more accurate counselling of patients regarding disease outcome, but also in paving the way for risk adapted therapy, since the prognostic value of certain markers varies according to which therapy the patient receives. This article summarises current concepts of prognostic markers in myeloma. 